\title{
EL DERECHO A LA VIDA Y EL DERECHO A LA PROTECCIÓN DE LA SALUD EN LA CONSTITUCIÓN: UNA RELACIÓN NECESARIA
}

THE RIGHT TO LIFE AND THE RIGHT TO HEALTH CARE IN THE CONSTITUTION: A NECESSARY RELATIONSHIP.

\author{
Alejandra ZÚÑIga FajuRI ${ }^{2}$ \\ Profesora de la Universidad de Valparaíso y \\ de la Universidad Diego Portales, Santiago, Chile. \\ alejandra.zuniga@uv.cl
}

RESUMEN: El presente trabajo analiza la relación que existe entre el derecho a la vida y el derecho a la protección de la salud a la luz de la doctrina, jurisprudencia y recientes reformas legislativas. Se razona en torno a las prácticas jurisprudenciales relativas a ambos derechos así como la influencia que, sobre el contenido del derecho a la protección de la salud en la Constitución, habría tenido la Reforma del AUGE del año 2005.

ABSTRACT:This paper analyzes the relationship between the right to life and the right to health care in relation with doctrine, jurisprudence and recent legislative reforms. Legal practice around both rights as well the influence of AUGE Health Care Reform allows analyze the content of the right to life and the relationship with health care right in the Constitution.

Palabras-Clave: Vida, equidad, cuidado sanitario, Constitución, AUGE.

KEYWORD: Life, fairness, health care, Constitution, AUGE.

\section{INTRODUCCIÓN}

El presente trabajo intentará definir la relación que existe entre el derecho a la vida y el derecho a la protección de la salud, tal y como se contemplan en nuestra Constitución y a la luz de la doctrina, jurisprudencia y recientes reformas legislativas. Se pretende reflexionar a partir de los dilemas relativos al concepto de derecho a la vida, a la luz de lo establecido tanto en las normas vigentes como en las prácticas jurisprudenciales de las cortes de protección que han conocido

\footnotetext{
${ }^{1}$ Este artículo forma parte del proyecto de investigación № 11080005, financiado por el Fondo Nacional de Desarrollo Científico y Tecnológico (FONDECYT), titulado: "Teorías de la justicia y Reforma Sanitaria AUGE".

${ }^{2}$ Doctora en Derecho. Profesora de derecho constitucional de la Escuela de Derecho de la Universidad de Valparaíso, Chile y de la Escuela de Derecho de la Universidad Diego Portales, Santiago, Chile. Enviado con fecha 23 de noviembre de 2010 y aprobado el 23 de marzo de 2011.
} 
recursos que sostienen la vulneración de este derecho en conexión directa con el derecho a la protección de la salud.

Junto con lo anterior, se reflexiona respecto de la consistencia de dichas prácticas jurisprudenciales con la concepción de derecho a la vida y a la protección de la salud sostenida por esa misma doctrina y jurisprudencia y por la definición que, de ambos derechos, se defenderá a lo largo de este artículo. En la misma línea, se pretende incorporar un nuevo análisis relativo a la influencia que la Reforma del AUGE del año 2005 y la reciente jurisprudencia de la Corte Suprema y del Tribunal Constitucional, habrían tenido sobre el contenido del derecho a la protección de la salud en la Constitución.

\section{1. ¿QUUÉ SIGNIFICA TENER DERECHO A LA VIDA?}

Esta compleja pregunta tiene, sin duda, implicaciones dogmáticas de la mayor importancia. Para comprender la tesis que se defenderá y el análisis que, en base a la misma, se hará de las normas y jurisprudencia nacionales, debemos comenzar recordando que el derecho a la vida, como todo derecho subjetivo, posee un piso y un techo que deben ser delimitados coherentemente. Frente a cualquier derecho reconocido en algún catálogo es indispensable, debido a la amplitud y abstracción con que éstos son consagrados, tener como instrumentos de interpretación ciertos principios que permitan echar luz, precisamente, sobre ese piso y ese techo que supone la exigibilidad de los derechos.

En este contexto, la pregunta clave parece ser qué significa decir que se tiene "derecho a la vida". Una autora que ha dado luces sobre este punto es Judith Jarvis Thomson, quien sostiene que el derecho a la vida puede entenderse fundamentalmente de tres maneras. En primer lugar, como un derecho que supone una obligación tanto negativa como positiva, es decir, por una parte, el derecho a no ser privado de la vida -a que nadie me mate- $y$, por otra, el derecho a recibir al menos lo mínimo indispensable para sobrevivir. En segundo lugar, algunos interpretan el derecho a la vida de modo más estricto y sostienen que éste no incluye el derecho a recibir algo por parte del Estado, sino que supone únicamente el derecho a que nadie atente contra mi propia vida. Por último, se sostiene que esa obligación de abstención admite, en verdad, excepciones como la legítima defensa o la pena de muerte, de manera que tenemos derecho tan sólo a que nadie nos prive arbitraria o injustamente de la vida ${ }^{3}$.

Según veremos, la jurisprudencia nacional, al resolver casos relativos al derecho a la vida y su relación con el derecho a la protección de la salud, varía su posición

${ }^{3}$ Thomson, J. J. (1980), pp. 211-240. 
respecto de la concepción adecuada del derecho establecido en el artículo $19 \mathrm{~N}^{\circ} 1$ de la Constitución, comprendiendo que, en ciertos casos, se trata de un derecho que genera tanto obligaciones positivas como negativas $y$, en otros casos, que se trata de una prerrogativa que sólo entrega a su titular la facultad de exigir que no se le prive, arbitrariamente, de la vida. En general, luego, la doctrina y jurisprudencia no consideran la segunda interpretación dada por Thomson.

Analicemos, entonces, la primera comprensión del derecho a la vida mencionada por esta filósofa: ¿ Tiene el Estado la obligación de conceder lo mínimo indispensable para mantener la vida de sus habitantes? Es posible encontrar respuesta a esta pregunta en el análisis de las normas constitucionales sobre la materia y, también, en el estudio de normas legales que son, finalmente, reflejo de políticas públicas estatales que, precisamente, parecen considerar que ambos derechos, cuidado sanitario y vida, están estrechamente vinculados. La Comisión de Estudios de la nueva Constitución, que sirven a los jueces subsidiariamente para interpretar sus normas, dejó prácticamente sin contenido el numeral $1^{\circ}$ del artículo 19 de la Constitución que asegura a todas las personas: "El derecho a la vida y a la integridad física y psíquica de la persona”. En efecto, la Comisión reflexionó únicamente sobre si el numeral debía incluir o no la prohibición de la pena de muerte y del aborto ${ }^{4}$ sin que hubiera mayor análisis sobre cuál era el contenido específico del derecho a la vida y cuál era la obligación del Estado a su respecto. Con todo, es importante poner de relieve que, en un principio, la Comisión tuvo el propósito de tratar, a continuación del derecho a la vida, el derecho a la protección de la salud, por estimarse que entre ambos derechos existía una estrecha vinculación ${ }^{5}$. Sin embargo, por razones metodológicas se abandonó ese plan ${ }^{6}$.

Puesto que, como hemos sostenido, la doctrina y jurisprudencia no consideran la segunda interpretación dada por Thomson, nos queda por analizar la tercera. ¿Supone el derecho a la vida sólo el derecho a que nadie nos prive arbitrariamente de la misma? Esa es, sin lugar a dudas, la posición mayoritaria de la doctrina nacional que, en general, rechaza las otras dos interpretaciones que consideran que la vida puede ser un derecho absoluto y, a su vez, que ella puede implicar para terceros -entiéndase, el Estado- una obligación positiva con el objeto de garantizar la mantención de la misma ${ }^{7}$.

\footnotetext{
${ }^{4}$ Actas oficiales de la Comisión de Estudios de la nueva Constitución, Sesión 84, 4 de noviembre de 1974.

${ }^{5}$ Actas oficiales de la Comisión de Estudios de la nueva Constitución, Sesión 87, p. 5 y sesión 90, p. 22.

${ }^{6}$ Pfeffer (1985), p. 368.

${ }^{7}$ Véase una revisión de la doctrina nacional sobre derecho a la vida en FIGUEROA, Rodolfo (2008).
} 
Para poder entregar una adecuada interpretación del derecho a la vida es necesario considerar su relación con otro derecho estrechamente vinculado: el derecho a la protección de la salud. Entre la doctrina nacional que se ocupa de examinar las relaciones existentes entre derechos ${ }^{8}$ se encuentra Germán Urzúa Valenzuela, quien sostiene, siguiendo a Hübner, que el derecho a la vida "no implica, evidentemente, tan sólo la facultad de impedir que se nos dé muerte, sino también la concurrencia de un conjunto de condiciones laborales, sociales, económicas, asistenciales y sanitarias que hagan factible el mantenimiento de la existencia dentro de un nivel propio de la dignidad humana. Representan, por lo tanto, aspectos o derivaciones de este atributo básico derechos tales como (...) el derecho a la protección de la salud"9. Otros autores, luego de reconocer que no hay referencia expresa, en las actas de la Comisión, del contenido global del numeral en estudio, señalan que "se infiere que el reconocimiento al derecho a la vida aparece referido preferentemente al soporte biológico y psíquico del hombre (...) el derecho a la vida representa, entonces, la facultad jurídica, o poder, de exigir la conservación y la protección de la vida humana, o sea, de ese estado de actividad sustancial propio del hombre"10.

Rodolfo Figueroa, por su parte, considera difícil una interpretación como esta pues se estaría equiparando el derecho a la vida con la salud ${ }^{11}$, tesis que, como veremos, confunde dos cuestiones que, precisamente, buscamos aclarar en estas líneas. Una comprensión adecuada y completa del derecho a la vida necesariamente supone también una concepción precisa de qué es el derecho a la protección de la salud, cuál es su contenido específico y qué relación es posible trazar entre ambos derechos.

Desde ya, a partir de la noción de derechos humanos se argumenta que el derecho a la vida debiera ser entendido como un derecho prioritario, en el sentido de que es un derecho que no admite, al momento de legitimar su supresión, la invocación de consideraciones de carácter económico o político. Así, cuando se usa la expresión derechos humanos se está hablando, generalmente, de aquellas facultades para cuya titularidad es condición necesaria y a la vez suficiente el pertenecer "a la clase de los seres humanos", excluyéndose así cualquier otro atributo idiosincrásico, biológico, institucional o socialmente adquirido que sea extraño o distinto al hecho de pertenecer a dicha clase ${ }^{12}$. Por ejemplo, el derecho a la vida o a la libertad es un derecho

${ }^{8}$ Por ejemplo, ni José Luis CEA ni Sergio Diez analizan una posible vinculación entre el derecho a la vida y el derecho a la protección de la salud. Cea (1999), Sergio Diez (1999).

${ }^{9}$ Urzúa Valenzuela (1991), pp. 179-180.

${ }^{10}$ Verdugo, Pfeffer y Nogueira (1994), pp. 192-3. Destacado agregado.

${ }^{11}$ Figueroa (2008), p. 274.

12 PeÑa (1992), p. 46. 
humano si y sólo si estamos dispuestos a reconocerlo a cualesquiera que sea miembro de esta clase, con prescindencia de toda otra consideración ${ }^{13}$.

El filósofo John Rawls ha sostenido que decir que los derechos humanos son absolutos significa que no son susceptibles de "negociación o regateo", o sea, no son susceptibles de ser puestos en una balanza para ser, eventualmente, desplazados por consideraciones extrañas a los propios derechos ${ }^{14}$. En caso de conflicto, entonces, los derechos humanos desplazan a cualquier otra consideración que no se refiera a derechos. En la misma línea, Ronald Dworkin argumenta que decir que los individuos tienen derechos supone darles reconocimiento aun cuando la mayoría o el gobierno piensen, incluso con buenas razones, que el beneficio social agregativo sería mayor de no existir tales derechos ${ }^{15}$. Por ejemplo, el beneficio social que se produciría al construir nuevas carreteras o al comprar aviones de guerra, en vez de utilizar esos recursos para garantizar el derecho a la protección de la salud. Ningún sentido tendría presumir que poseemos derechos si no estamos dispuestos a conceder que ellos existen incluso contra el beneficio general. De esa manera, los derechos humanos operan como restricciones al bien común y como test de legitimidad de las políticas públicas, constituyéndose así como límite fundamental frente a las acciones u omisiones arbitrarias o ilegales del poder público.

En fin, es posible estimar que el derecho a la vida incluye e incorpora necesariamente algún ámbito o esfera de protección del derecho a la protección de la salud que significa, a lo menos, asegurar aquellas prestaciones mínimas de las cuales depende directamente la vida de las personas, y que esa esfera de protección es absoluta en el sentido de que no admite pretextos de orden patrimonial.

\section{El DERECHO A LA PROTECCIÓN DE LA SALUd en la Constitución de 1980 antes del AUGE}

La Constitución regula el derecho a la protección de la salud en el artículo $19 \mathrm{~N}^{\circ}$ 9, imponiendo al Estado la obligación de resguardar el libre e igualitario acceso a las acciones de promoción, protección y recuperación de la salud y de rehabilitación del individuo, así como la coordinación y control de las acciones relacionadas con la salud. Reconoce, a continuación, el derecho a elegir el sistema de salud, estatal o privado, al que acogerse, siendo este último inciso el único que podría garantizarse por la vía judicial, a través del Recurso de Protección. A

\footnotetext{
${ }^{13}$ Sobre los dilemas morales que es necesario resolver para definir a quienes considerar como miembros de la "clase de los seres humanos", véase VillavicEncio, Luis $(2008,2010)$.

${ }^{14}$ JoHn RaWLS (1971).

${ }^{15}$ Ronald Dworkin (1989), pp. 276-303.
} 
primera vista, entonces, podría sostenerse que se contempla a la atención sanitaria sólo como un derecho negativo que implica la mera libertad de adquirir asistencia en el mercado de la protección de la salud, puesto que el único inciso que está garantizado judicialmente es aquel que reconoce el derecho a "elegir el sistema de salud al que desee acogerse, sea éste estatal o privado" 16 . Esto supone entender que el constituyente no habría consideración a la "necesidad" de atención médica -en la línea de las legislaciones comparadas y de las teorías de la justicia igualitaristas- como condición suficiente para recibir dicha atención sino que, por el contrario, se coloca a la capacidad de pago como único fundamento para acceder a los recursos sanitarios, lo que contraría las pretensiones de equidad propias de todo estado constitucional moderno.

Antes de la Reforma sanitaria AUGE, las normas que reglamentaban y desarrollaban el derecho a la protección de la salud garantizaban la entrega de prestaciones mínimas exclusivamente para salvaguardar la vida. El caso paradigmático en este sentido lo constituye la Ley No 18.469, que "Regula el ejercicio del derecho constitucional a la protección de la salud", y su modificación por la Ley No 19.650, el año 1999, que estableció para todos los centros médicos del país la prohibición de exigir dinero, cheques u otros instrumentos financieros para avalar el pago o condicionar de cualquier otra forma una "atención médica de urgencia" ${ }^{17}$. Para los demás casos, la regla era la prestación del servicio de forma limitada, de modo que las omisiones o negativas del Estado a entregar atención médica no se estimaban, por los tribunales, arbitrarias o ilegales, puesto que se habría condicionado fuertemente el otorgamiento de las prestaciones de salud. En efecto, argumentaba la jurisprudencia, "la Ley $\mathrm{N}^{\circ} 18.469$, que regula el derecho constitucional a la protección a la salud y crea un régimen de prestaciones de salud, señala en su artículo 11 que las prestaciones comprendidas en el régimen "se concederán por esos organismos a través de sus establecimientos, con los recursos físicos y humanos de que dispongan"18. Luego, se entendía que se trataba de un derecho que no poseía contenido constitucional propio y que estaría falto de garantías.

A resultas de lo anterior, se presentaron numerosos recursos de protección destinados a exigir directamente la garantía del derecho a la protección de la salud contemplado en el inciso primero del artículo $19 \mathrm{~N}^{\circ} 9$ que, como se explicó, reconoce a todas las personas, sin más, "el derecho a la protección de la salud".

\footnotetext{
${ }^{16}$ Artículo 19 N 9 inciso final y artículo 20 de la Constitución Política de 1980.

${ }^{17}$ La modificación legal a la Ley 18. 469 dispone que en los casos de urgencias médicas: "se prohíbe a los prestadores exigir a los beneficiarios de esta ley, dinero, cheques u otros instrumentos financieros para garantizar el pago o condicionar de cualquier otra forma dicha atención".

${ }^{18}$ Corte Suprema, Rol № 4408-00. Santiago, trece de diciembre del año 2000.
} 
Como se sabe, el principio de aplicación directa de los derechos fundamentales implica que su eficacia es independiente de la existencia o no de una ley que los regule y que son derechos que deben ser reconocidos de oficio por parte de los servidores públicos, en particular, de los jueces. En el Estado Constitucional, los derechos son atribuciones, facultades o potestades independientes de la legislación común, puesto que adquieren valor jurídico de la propia Constitución y los instrumentos internacionales. Por ello, son perfectamente aplicables de modo directo, aun sin la existencia de normas inferiores que los regulen y desarrollen. Así, se hace necesario distinguir entre "la ley como regla establecida por el legislador, y los derechos humanos, como pretensiones subjetivas absolutas, válidas por sí mismas, con independencia de la ley" ${ }^{19}$. En ese mismo sentido, Bidart sostiene que la fuerza normativa que singulariza la Carta Fundamental, "apareja necesariamente que ella está dotada de fuerza normativa para operar sin intermediación alguna, y obligación (para todos los operadores gubernamentales y para los particulares en sus relaciones 'inter privatos') de aplicarla, cumplirla, conferirle eficacia, no violarla-ni por acción ni por omisión”20.

Otra importante consecuencia judicial que se pudo observar a propósito de la mencionada regulación legal, se generó al aumentar la interposición de demandas en busca del reconocimiento del derecho a la protección de la salud, no directamente esta vez, sino que por medio de considerar su evidente relación con el derecho a la vida o el derecho a la igualdad ante la ley, que sí estarían garantizados por el Recurso de Protección. El mandato constitucional obligaría a los tribunales a desarrollar una interpretación jurisprudencial que haga reconocible y exigible el derecho a la protección de la salud por medio de una definición amplia que permita identificar un contenido constitucional propio o, al menos, un reconocimiento indirecto ${ }^{21}$.

Por último, la interpretación restringida del derecho a la protección de la salud tuvo consecuencias sociales significativas, pues sería uno de los elementos que determinaron la consolidación de los preocupantes resultados que Chile presentaba al año 2000 en la materia, según los datos de la Organización Mundial de la Salud. En efecto, se revelaba la existencia de serios problemas de discriminación y equidad asociados a los altos índices de desigualdad tanto en el acceso a los servicios básicos como en los deficientes resultados de los indicadores nacionales que se expresan, entre otros, en diferencias significativas en la esperanza de vida y

${ }^{19}$ ZaGRebelsky (2002), p. 47.

${ }^{20}$ Bidart Campos, 1995 , pp. 88-89.

${ }^{21}$ Sobre las estrategias judiciales que se han desarrollado en la jurisprudencia comparada para hacer justiciables, por la vía de la demanda directa o indirecta, los derechos sociales, véase ABRAMOVICH y Courtis (2002). 
en la discapacidad. En los índices de desarrollo humano -en el ítem "equidad del sistema sanitario"-, Chile aparecía con una de las peores evaluaciones del mundo, pues se tomó en consideración tanto la carga financiera que representa la salud para las familias, como la desigualdad en la calidad y oportunidad de respuesta que ofrece el sistema ${ }^{22}$.

\section{JURISPRUDENCIA NACIONAL: LA RELACIÓN INCIERTA ENTRE LOS DERECHOS}

En marzo del año 1999, José Gabriel Sierra Banda, joven de 25 años de edad con VIH-Sida, presentó un Recurso de Protección con el objeto de obligar al Hospital San José a adquirir los medicamentos -la triterapia- que requería para seguir con vida, argumentando que todos los estudios, nacionales e internacionales, sobre el VIH-Sida coinciden en que interrumpir la terapia antirretroviral implica un alto riesgo de muerte ${ }^{23}$. $\mathrm{Al}$ momento de la presentación del recurso, el estado de salud de Gabriel era tan grave que su nivel de CD4 en el organismo era de cero por $\mathrm{mm} 3$ de sangre, es decir, su sistema inmunológico estaba totalmente paralizado $^{24}$. Los otros recurrentes, Luis Vivanco González y Anthony García López, se encontraban en una situación médica similar a la de Sierra, de manera que la demanda ante la Corte de Apelaciones de Santiago se fundamentaba en la violación del artículo $19 \mathrm{~N}^{\circ} 1$ de la Constitución, así como también, de las normas respectivas de diversos tratados internacionales de derechos humanos.

Se sostenía, en el recurso, que el derecho a la vida no sólo importa la obligación general de omitir actos atentatorios contra la misma, sino que, además, comprende un deber de velar porque dicha vida pueda desarrollarse bajo el amparo del Estado, lo que implica la obligación general para todos los sujetos de no atentar contra la vida de otros y, además, la obligación específica y concreta de la autoridad de velar positiva y activamente por el normal y pleno desarrollo de la misma, cuando ella se ve amenazada o perturbada por actos de terceros o por hechos naturales. Como puede apreciarse, esta interpretación del derecho a la vida se acerca a la

\footnotetext{
${ }^{22}$ En materia de distribución de la carga financiera en salud, Chile estaba ubicado en el lugar 168 de entre 191 países considerados. Informe sobre la Salud en el Mundo 2000: Mejorar el desempeño de los sistemas de salud. OMS. Ginebra. 2000.

23 "Declaración sobre el uso de terapia antirretroviral en pacientes infectados por virus de inmunodeficiencia humana", Comité Consultivo del Sida, Revista Chilena de Infectología (1998), pp. 183-187.

${ }^{24} \mathrm{El} \mathrm{VIH}$, como todo virus, infecta una célula y utiliza su maquinaria para replicarse (multiplicarse). El VIH ataca las células de CD4 o linfocitos T4, coordinadores de las defensas y parte esencial de nuestro sistema inmunológico. Los protocolos clínicos establecen que cuando una persona tiene menos de 300 CD4 por mm3 de sangre, está en situación de desarrollar el Síndrome de Inmunodeficiencia Adquirida (Sida), puesto que su sistema inmunológico está debilitado y es probable que surjan las llamadas "enfermedades oportunistas" que coactúan para configurar el Síndrome de Inmunodeficiencia Adquirida.
} 
propuesta en primer lugar por Thomson: derecho que acarrea tanto obligaciones negativas como positivas ${ }^{25}$.

Los siguientes recursos invocaron el Decreto Supremo No 362 del Ministerio de Salud, en el cual se incluía expresamente al Sida dentro de la lista de enfermedades de transmisión sexual respecto de las cuales los Servicios de Salud dependientes del Estado estaban obligados a entregar tratamiento y control completos y totalmente gratuitos. Los tribunales de justicia, sin embargo, rechazaron nuevamente las demandas, bajo la tesis de que éste no era un caso en el que estuviera implicado el derecho a la vida, sino que, más bien, el derecho a la protección de la salud (no amparado por la acción de protección). "Cabe precisar, desde ya, que en los recursos acumulados se formulan peticiones basadas en el 'derecho a la vida', aludiendo al numeral $1^{\circ}$ del artículo 19 de la Carta Fundamental, que precisa la protección a la vida del que está por nacer, la restricción de la aplicación de la pena de muerte y la prohibición de aplicar apremios ilegítimos, o sea, su alcance alude a conductas que pongan en peligro la vida humana, sin que pueda estimarse como tales las omisiones que se atribuyen a los Servicios de Salud y al Ministerio respectivo, puesto que el peligro a la vida de los recurrentes deriva de la enfermedad de que, lamentablemente, padecen pero no de las autoridades sanitarias" 26.

La dificultad lógica de intentar distinguir de manera estricta entre acción y omisión, como causa de un resultado, ha sido ampliamente estudiada ${ }^{27}$. No se pretende ignorar el hecho evidente de que los recurrentes estaban muriendo de Sida y, por ello, de un hecho natural. Sin embargo, ¿tenía esto alguna importancia a la hora de comprobar la omisión ilegal y arbitraria del Estado? Admitir este razonamiento resulta tan absurdo como sostener que si un padre no otorga alimento

${ }^{25}$ Como el recurso fue declarado inadmisible -y, por tanto, se agotaron los recursos internos- se presentó una medida cautelar ante la Comisión Interamericana de Derechos Humanos, logrando que el Estado de Chile accediera a entregar los medicamentos requeridos a los tres recurrentes (artículo 64 de la Convención Americana de Derechos Humanos).

${ }^{26}$ Corte Suprema, Rol No 4408-00. Santiago, trece de diciembre del año 2000. Considerando $14^{\circ}$. Destacado agregado.

${ }^{27}$ La discusión sobre la causa primera de un cierto resultado, clásica en el Derecho penal, se ha profundizado a propósito de los dilemas bioéticos asociados a los derechos del paciente y la eutanasia. ¿¿s distinto no iniciar un tratamiento que interrumpirlo? Muchos piensan que la omisión es menos causa de lo que por ella acontece y que es moralmente peor iniciar la cadena causal que tiene como resultado un daño (actuar) que no interrumpirla si ya ha empezado (omitir). Con todo, no es fácil explicar la diferencia entre el médico que busca, por piedad, matar al enfermo y el otro que, para aliviar su sufrimiento, le proporciona una droga consciente de que la muerte puede producirse o acelerarse a consecuencia. ¿De qué manera, se pregunta Frey, el médico podría ser causa parcial de esa muerte? Si administra una dosis alta de morfina que produce una depresión respiratoria y el enfermo muere, sería falso atribuir su muerte a un fallo respiratorio sin indicar lo que lo provocó, como lo sería achacar la muerte a un individuo al que le pasó un tren por encima sin indicar que alguien lo empujó a la vía. Véase ZúNíigA F., Alejandra, 2008a, pp. 111-130. 
a su hijo éste sólo muere por inanición y no, también, por la inacción del padre; o pretender que cuando un paciente recurre de urgencia por un accidente a un hospital y éste se niega a atenderlo, no habrá fallecido debido a la omisión de atención del hospital, sino que debido sólo a la acción del automóvil.

En fin, la argumentación de los tribunales parece dar por supuesto que el único derecho comprometido en estos casos es el derecho a la protección de la salud. Pero ¿es posible sostener que la afectación del derecho al cuidado sanitario puede transformarse en la violación del derecho a la vida? Parece, entonces, necesario reflexionar sobre la relación directa y esencial que puede llegar a existir entre vida y salud. Desde ya, dicha conexión fue reconocida por el legislador al modificar el artículo 11 de la Ley 18.469, que prohíbe negar atención médica en casos de urgencia vital. En efecto, se consideró que la emergencia vital debía siempre ser atendida, pues en esas situaciones la relación salud-vida era evidente. Ahora, ¿cuándo se está ante un caso de "urgencia" médica? ¿Qué grado de inminencia debe tener la muerte a consecuencia de una emergencia médica, minutos, horas o días? ¿Es esa una distinción -la horaria- razonable o debiera bastar tan sólo la inminencia de la muerte a resultas de la falta de atención?

Revisemos más jurisprudencia para dar luces sobre este asunto. Varios particulares recurrieron de protección, en julio de 1993, contra la instalación de un servicentro para el expendio de combustible que se ubicaría en Cerro Alegre de Valparaíso y que ocasionaría contaminación. En ese caso, la Corte Suprema consideró que, "efectivamente aparece amenazado el derecho a la vida y la integridad física de los recurrentes, sus familiares y habitantes del barrio, lo cual ordena proteger la Constitución Política de la República en el artículo 19 No 1, en armonía con su artículo 20 y conduce a los sentenciadores a acoger el presente recurso" 28 . Ahora, siguiendo con la línea argumental iniciada, podemos preguntarnos, ¿̨cuál era la inminencia del daño en un caso como ese? De un modo similar habría razonado la Corte de Apelaciones de Santiago cuando dos abogados de la Vicaría de la Solidaridad interpusieron un recurso de protección debido a que estaban recibiendo amenazas de muerte, sosteniéndose que "en estas condiciones y teniendo presente que estas amenazas a la vida e integridad física y psíquica son justos temores (...) y como el Estado a través de su organización o autoridades correspondientes debe resguardar la seguridad nacional dando la adecuada protección a las personas, protegiéndolos de actos arbitrarios o ilegales, como son los que agravian, perturban o amenazan a las personas por quienes se recurre, que dicen relación con el ejercicio legítimo de la garantía constitucional que ampara el No 1 del artículo

${ }^{28}$ Revista Fallos del Mes, № 415, Santiago, 1993, p. 360. 
19 de la Constitución Política, que es el derecho a la vida y a la integridad física y psíquica de las personas, procede acoger este recurso"29.

La decidida protección que se entrega al derecho a la vida en estos casos se extiende frente a situaciones en las que, en principio, también está involucrado el derecho a la protección de la salud y que, por razones que no son claras, los tribunales han decidido en forma diversa a como lo hicieron en los casos de VIHSida mencionados. Así, en 1992, el director del Hospital de Copiapó, Omar Luz Hidalgo, recurrió de protección con el objeto de que se realizara, contra de la voluntad del paciente de 20 años Luis Muñoz Bravo, perteneciente a la religión de la Congregación Testigos de Jehová, las transfusiones de sangre necesarias para salvarle la vida. Tanto la Corte de Apelaciones de Copiapó como la Corte Suprema, en su sentencia de confirmación, resolvieron que: "En consideración a la entidad del bien jurídico cuya protección se solicita y la necesidad urgente que existiría de cautelarlo, se ordena oficiar al señor Director del Hospital para que éste o quien lo subrogue disponga se aplique aun en contra de la voluntad de don Luis Bravo o de sus familiares, la terapia que sea necesaria para el tratamiento de la enfermedad que éste padece, incluida la transfusión sanguínea, pudiendo para ello recabar el auxilio de la fuerza pública en caso de oposición, debiendo informar a esta Corte las medidas que adopte", agregando que entre "el cotejo de los intereses en conflicto, el derecho a la vida y la libertad de conciencia, debe prevalecer el primero de los nombrados, porque la recurrida solicitó la atención médica para su hijo, a fin de preservar su salud y conservar su vida, disponiendo el hospital de los elementos humanos y materiales para que el paciente recuperara la salud y mantuviera la vida, proporcionándosele al enfermo los auxilios médicos necesarios, entre ellos la transfusión de sangre, que lo han llevado a su recuperación. Que, por otra parte, el juramento y rol profesional del médico lo obligan a preservar la vida" ${ }^{\prime 0}$.

En la sentencia de protección presentada a favor de Jorge Omar Reyes Ibarra, quien se encontraba internado en estado de gravedad debido a que se habían negado terminantemente, él y su madre, a que se le efectuaran transfusiones de sangre por ser Testigo de Jehová, la Corte de Apelaciones de Rancagua -cuyo fallo fue confirmado por la Corte Suprema el 22 de agosto de 1995- acogió el recurso al considerar que: "Constituye una obligación de los médicos tratantes de la persona en cuyo favor se ha incurrido, el procurar por todos los medios y técnicas que integran la lex artis médica el mantener la vida de sus pacientes (...) en razón de que debe primar la preservación de la salud y la vida de las personas sobre cualquiera otra consideración aunque sea de índole religiosa que ponga en

${ }^{29}$ Revista Gaceta Jurídica, N 59, Santiago, 1985, p. 46-49.

${ }^{30}$ Revista Fallos del Mes, № 402, Santiago, 1992, pp. 227-231. 
riesgo innecesariamente la vida del enfermo". La Corte continuó su razonamiento sosteniendo que: "la vida se garantiza en la Constitución en la medida que pueda privarse de ella al individuo por agentes extraños a él, por un atentado de terceros, resultando evidente que se encontraba el paciente seriamente amenazado, por la actitud de la recurrida, en el derecho a la vida y a la integridad física y síquica, puesto que de persistirse en su planteamiento se puede inferir el progresivo deterioro en la salud y un posible desenlace fatal de no otorgarse el tratamiento aconsejado por su médico, arriesgándose innecesariamente la vida del enfermo" ${ }^{31}$. Similar razonamiento ha tenido la Corte Suprema en los casos de Matías Silva ${ }^{32}$, Patricia Castillo ${ }^{33}$, y en el caso de huelga de hambre de Patricio Ponce y otros ${ }^{34}$, acogiéndose en todos ellos los recursos de protección presentados.

En las sentencias relatadas, los tribunales superiores de justicia han razonado desde la consideración de que el derecho a la vida, cuando se ve afectado como consecuencia de una enfermedad posiblemente fatal, tanto los médicos como las instituciones de salud involucrados, tienen el deber de hacer todo lo humanamente posible para que la salud del paciente sea preservada y, con ello, su derecho a la vida, bastando entonces como fundamento para dar protección, "la entidad del bien jurídico cuya protección se solicita y la necesidad urgente que existiría de cautelarlo" ${ }^{35}$. Dicho de otro modo, en estos casos, los tribunales parecen comprender de manera amplia el derecho a la vida-siguiendo a Thomson-advirtiendo que se trata de un derecho que incorpora tanto deberes de acción como de omisión. Sin embargo, este intachable razonamiento cambia abruptamente cuando se involucran grandes recursos del Estado, como ocurre en los casos a favor de personas con VIH-Sida y, también, en el caso de Olga Gallardo Soto, quien recurrió de protección en contra del director del Servicio de Salud Metropolitano Oriente, pues éste se había negado a proporcionarle el tratamiento de hemodiálisis sin el cual, como se constataba en autos, ella moriría irremediablemente en el breve plazo. Similar destino tendría Miguel Serey a consecuencia de la sentencia de la Corte Suprema que, confirmando el fallo de primera instancia, consideró que no se atentaba contra su derecho a la vida al ponerlo en lista de espera para recibir la

${ }^{31}$ Revista Gaceta Jurídica, № 50, Santiago, 1984, p. 76.

${ }^{32}$ Revista Gaceta Jurídica, № 232, Santiago, 1999, pp. 107-108.

${ }^{33}$ Corte Suprema, Rol No 33.945, 18 de enero de 1996.

${ }^{34}$ El caso de Patricio Ponce y otros se presentó por las autoridades de la Universidad Católica, quienes solicitaban a la Corte que obligara a los estudiantes a abandonar la huelga de hambre por ellos iniciada, puesto que atentaría contra el derecho a la honra de las autoridades de la universidad y a la vida de los huelguistas. Revista Gaceta Jurídica No 50, Santiago, 1984, pp. 76-79.

${ }^{35}$ Revista Fallos del Mes, № 402, Santiago, 1992, pp. 227-231. 
atención urgente de hemodiálisis ${ }^{36}$. Es decir, a pesar de que, como en el caso de los Testigos de Jehová, ambos recurrentes se encontraban en una situación en que "se puede inferir el progresivo deterioro en la salud y un posible desenlace fatal de no otorgarse el tratamiento aconsejado por su médico" ${ }^{37}$, los tribunales decidieron no entregarles la protección demandad $a^{38}$. Mientras la Corte discurrió que las enfermedades sufridas por personas pertenecientes a la religión de los Testigos de Jehová sí afectaban su derecho a la vida y debía, a toda costa, ser resguardado por el Estado, ello no ocurría con la enfermedad de la señora Gallardo o de Miguel Serey, aun cuando fue probado en autos que de no recibir urgentemente la atención médica requerida morirían en el breve plazo.

Para los tribunales chilenos, entonces, el hecho de que esté en juego la vida no significa que esté involucrado el derecho a la vida contenido en la Constitución. El Ministro Osvaldo Erbetta concurrió a la revocatoria del fallo de primera instancia y al rechazo del recurso, pero no aceptó los fundamentos de la sentencia, pues, "el caso de la señora María Olga Gallardo Soto ya dejó de ser un asunto de salud, respecto del cual no procede el recurso de protección, sino uno de vida, que es un bien protegible según el artículo 20 de la Constitución (...). No obstante, tal como se establece en algunos fundamentos de este fallo, que se aceptan, debiendo el Servicio Público estarse a su presupuesto (...) no ha habido por su parte infracción a la ley ni arbitrariedad al negar una prestación de servicio que le era imposible dar, lo que conlleva al rechazo del recurso (...). No obstante lo resuelto, este Tribunal recomienda al Servicio recurrido que, dentro de sus posibilidades, siga prestando la atención médica que hasta ahora se ha dado a la paciente" ${ }^{\prime 39}$. Así, Erbetta estimó que no era posible, de los hechos de la causa, desconocer que era el derecho a la vida el que estaba comprometido. Sin embargo, consideró que, no obstante ello, ese derecho debía ser amparado sólo en tanto el Estado contase con recursos suficientes.

A la interpretación mayoritaria se opuso tajantemente el Ministro Servando Jordán, quien sostuvo que: "Es un hecho de esta causa que si la recurrente, de escasos recursos económicos y que padece de una nefropatía crónica, no recibe tratamiento mecánico de hemodiálisis, fallecerá a corto plazo; la realidad antedicha importa, de manera cierta, una directa e inmediata amenaza a la vida de esa paciente, por parte del Servicio de Salud Metropolitano Oriente, quien niega la urgente asistencia re-

\footnotetext{
${ }^{36}$ Revista Gaceta Jurídica, № 101, Santiago, 1988, pp. 21-22.

${ }^{37}$ Sentencia Corte Suprema Rol No 33.945, 18 de enero de 1996.

${ }^{38}$ Revista Gaceta Jurídica, № 91, Santiago, 1998, pp. 11-14.

${ }^{39}$ Ibíd, Revista Gaceta Jurídica, № 91. Destacado agregado.
} 
querida por la recurrente señora Gallardo, pretextando insuficiencia presupuestaria para dichos efectos y otras prioridades (...) en concepto del disidente, no parece valedera la afirmación de la parte recurrida, en cuanto a que sólo se vulnera la vida cuando existen actos ilegítimos y concretos que atenten contra ella, y que en la especie, existe sólo un problema relacionado con la salud de la recurrente, y ello no parece atendible, supuesto que en el caso se evidencia un curso orgánico terminal vinculado directamente con la cesación de la vida por omisión, y no una realidad que tenga atingencia con la mantención de una normalidad fisiológica, con la consecución de las potencias físicas o psíquicas ordinarias de un sujeto, o con la reobtención de un estado calificado como de salud" 40 . Dicha interpretación implica reconocer la obligación para el Estado en el primer sentido apuntado por Thomson, no sólo de no privar arbitrariamente de la vida a las personas, sino también, de proporcionarles lo mínimo indispensable para mantener la vida.

Probablemente, las conclusiones de los tribunales superiores en los casos relatados se sustenten en la conocida clasificación de los derechos humanos entre derechos civiles y políticos, inmediatamente exigibles, y derechos económicos, sociales y culturales, no susceptibles de protección debido a su supuesto carácter programático. La discusión en torno al estatus jurídico de los DESC se sustenta, en parte, en el artículo $2^{\circ}$ del Pacto Internacional de Derechos Económicos, Sociales y Culturales, que establece la llamada cláusula de "progresividad" y sostiene que los derechos de índole económico, como el derecho a la protección de la salud, admitirían una concreción gradual. Así, se ha sostenido que los derechos civiles y políticos, como el derecho a la vida, serían derechos de exigibilidad inmediata para el Estado, en cambio los DESC, como el derecho a cuidado sanitario, exigirían de parte del Estado una prestación que, por lo mismo, sólo es posible demandar gradualmente a los Estados en atención a sus particulares niveles de desarrollo. Con todo, lo anterior no significa que sea imposible identificar un núcleo minimo inmediatamente exigible a los Estados en relación con cada uno de los DESC. Es lo que, según veremos, ha hecho Chile con la Reforma Sanitaria AUGE.

Por cierto que dicha clasificación, en gran medida abandonada por la doctrina y jurisprudencia comparada e internacional (como nos recuerda Nogueira ${ }^{41}$ ), desconoce que derechos como el derecho a la vida y el derecho a la salud están íntimamente relacionados, llegando incluso en ciertas circunstancias a ser imposible la distinción entre uno y otro, como se deriva de la reforma a la Ley $\mathrm{N}^{\circ} 18.469$ analizada más arriba. ¿Podemos siempre saber claramente cuándo se está ante la violación del derecho a cuidado sanitario y cuándo se trata del derecho a la vida?

${ }^{40}$ Ibíd, Revista Gaceta Juridica, № 91.

${ }^{41}$ NogueIra (2009), pp. 143-205. 
Posiblemente no. Es necesario reconocer que, a veces, se están violando ambos derechos y que uno de ellos no admite argumentos de progresividad.

Finalmente, vale la pena revisar el fallo del 28 de agosto de 2001, redactado por el ministro Carlos Cerda, que acoge los recursos de protección presentados a favor de tres personas viviendo con VIH-Sida. Dicho recurso fue acogido en base al siguiente razonamiento: "Que, para analizar si la inacción de los recurridos se encuentra ajustada a derecho o si por el contrario es ilegal o arbitraria, se deben tener presentes los siguientes principios: a) de acuerdo a lo establecido por el inciso $4^{\circ}$ del artículo $1^{\circ}$ de la Constitución Política de la República de Chile el Estado está al servicio de la persona humana y su finalidad es promover el bien común. Por su parte, el artículo 19 No 1 de la Constitución Política de la República, asegura a todas las personas el derecho a la vida”. En primer lugar, entonces, la Corte de Apelaciones resolvió dejar de lado, inmediatamente, cualquier argumentación que desconociera el hecho cierto de que el recurso (al igual que en el caso de la señora Gallardo y del señor Serey) involucraba, por sobre cualquier otro derecho, al derecho a la vida.

Continúa el tribunal argumentando en base al principio de aplicación directa de los derechos fundamentales, que "siendo la vida un derecho inherente a la persona humana, es decir, un derecho de la personalidad que la doctrina ha reconocido existir por su propio valor moral, con independencia de que el legislador lo haya proclamado (...) tal derecho, debe ser respetado por todos, y en particular, por quien ha declarado solemnemente garantizar o asegurar a todas las personas 'el derecho a la vida' (...). Que, analizando la inminencia de la muerte de los recurrentes (hecho anunciado por los profesionales de la salud) en caso de no recibir tratamiento antirretroviral no es posible aceptar que, quien está al servicio de la persona humana y ha asumido, entre muchas otras políticas de Estado, la obligación de combatir las enfermedades de transmisión sexual -entre las que se encuentra expresamente considerado el Sida- observe o contemple sin intervenir cómo, precisamente, esas mismas personas a quienes asegura el derecho a la vida, la pierden". De igual forma, ante la objeción del Consejo de Defensa del Estado, de que los servicios públicos de salud no tenían recursos suficientes como para velar por la vida de los demandantes, la Corte sostuvo: "Que en relación a la falta de recursos: esta argumentación no es aceptable por cuanto, como ya se señaló, el derecho a la vida es un derecho de carácter absoluto y al margen de toda posibilidad de negociación patrimonial. El establecer un orden de prioridad para que los portadores de inmunodeficiencia humana, $(\mathrm{VIH})$ accedan al tratamiento farmacológico que les permitirá vivir, basado en razones técnicas pero determinado al fin por razones económicas, es jurídica y moralmente inaceptable pues establece, necesariamente, una discriminación arbitraria entre personas que se 
encuentran en una misma situación”“泣. Luego, cuando lo que está en juego es la vida, los criterios económicos no son admisibles, como no lo serían, tampoco, la violación de derechos políticos, del debido proceso, la libertad de expresión, etc. debido a la falta de recursos.

La Corte Suprema revocó la sentencia declarando que la omisión de los recurridos no era ni arbitraria ni ilegal, pues se trataba sólo del derecho a cuidado sanitario, sin explicar por qué, a su entender, el derecho a la vida no estaría comprometido ${ }^{43}$. En definitiva, puesto que el elemento 'recursos' jugaba nuevamente un papel preponderante, se consideró que este no era un caso de vulneración del derecho a la vida, aun cuando de los informes médicos y demás pruebas presentadas resultaba muy difícil concluir otra cosa, al igual que en los casos Gallardo, Serey, Testigos de Jehová, huelga de hambre y demás personas con Sida que durante tres años recurrieron de protección.

Finalmente, sólo resta referir que la sentencia del Tribunal Constitucional de 18 de abril de 2008 sobre la contracepción de emergencia (veremos en el apartado siguiente aquellas relativas al derecho a la protección de la salud) ha avalado la posición mayoritaria de la doctrina en relación con la interpretación del derecho a la vida en el tercer sentido apuntado por Thomson: sólo como el derecho a que nadie nos prive arbitrariamente de la vida ${ }^{4-45}$.

\footnotetext{
${ }^{42}$ Corte de Apelaciones, Rol No 3.025-2.001. Sentencia de 28 de agosto de 2001.

${ }_{43}$ Corte Suprema, Rol No 3.599-2001, recogiendo el considerando No 8 del fallo de la Corte de Apelaciones.

${ }^{44}$ Véase Nogueira, Humberto (2008), y también, Tórtora, Hugo (2005).

${ }^{45}$ No puedo dejar de anotar aquí que dicha sentencia, que se ocupa de definir el sentido y alcance del artículo $19 \mathrm{~N}^{\circ} 1$ concluyendo que, bajo el alero de la Constitución, "el que está por nacer" es persona, se equivoca gravemente.

Así, en primer lugar, la sentencia pretende obviar que, a la luz de la Constitución, sólo son personas -y por ello titulares de derechos- los seres humanos nacidos, puesto que el artículo que se interpreta habría reservado un inciso para las personas y otro distinto para el nasciturus. De seguir la tesis del Tribunal Constitucional, resulta inevitable preguntarse qué sentido habría tenido incorporar el inciso segundo del artículo 19 número 1 (cuestión a la que, precisamente, se opuso Jaime Guzmán).

En segundo lugar, esta comprensión, si se quiere, "gramatical" de la norma, coincide con su interpretación lógica y sistemática, puesto que el resto del ordenamiento jurídico nacional, tanto en el ámbito civil como penal, considera como estatutos diferenciados aquellos destinados a regular a las personas nacidas, por una parte, y al nasciturus, por otra (véanse las normas pertinentes del Código civil, a propósito del inicio de la existencia legal de las personas, y del Código penal, que distingue -y sanciona de manera diferente- el homicidio y el infanticidio).

En tercer lugar, sin perjuicio de que el mecanismo originalista no nos parece el más idóneo para interpretar la Constitución, es inevitable recordar aquí que la incorporación del mencionado inciso segundo - según consta en las Actas oficiales de la Comisión de Estudio de la Nueva Constitución, Sesión 87a de 14 de noviembre de 1974- tuvo precisamente como objetivo dejar claro que la mayoría de los redactores de la Constitución no quiso prohibir el aborto en todos los casos, sino que, cosa distinta, permitirlo en, por ejemplo, el supuesto de violación o en el caso de aborto terapéutico.
} 


\section{El DERECHO A LA PROTECCIÓN DE LA SALUd DESPUÉS DE LA REFORMA SANITARIA:}

Resulta importante analizar qué ha ocurrido con el derecho a la protección de la salud después de la Ley AUGE, No 19.966 (promulgada el 25 de agosto de 2004), que estableció un Régimen de Garantías en Salud para asegurar a todas las personas residentes en el territorio nacional, "independiente de su edad, sexo, educación, etnia, preferencias sexuales o ingreso", el acceso universal a una atención de salud adecuada y oportuna para enfrentar las situaciones de enfermedad. ${ }^{46}$ ¿Qué implicancias tiene el AUGE desde el punto de vista constitucional? Sabemos que como no todos los servicios sanitarios son igualmente importantes (una parte salva vidas, mientras que otra sólo mejora la calidad de vida) debemos responder a la cuestión relativa a qué necesidades sanitarias debemos satisfacer cuando no podemos cubrirlas todas. Una respuesta posible y justa (avalada por la OMS y por los mejores sistemas sanitarios del mundo) es la que entrega el actual Sistema Nacional de Salud que, con la reforma del AUGE, garantiza la cobertura de un cierto número de patologías - definidas como prioridades sanitarias al ser los problemas de salud que causan mayor mortalidad y morbilidad en Chile- de modo universal.

Como se sabe, la Reforma AUGE obliga a los prestadores públicos (FONASA) y privados (ISAPRES) a proporcionar planes de salud construidos sobre la base de ciertas prioridades sanitarias con las siguientes garantías explícitas: a) Garantía de acceso, conforme a la cual todos los individuos deberán recibir atención y ser parte de una red de salud en su lugar de residencia; b) Garantía de oportunidad, según la cual habrá un límite máximo de tiempo preestablecido para asegurar tanto la primera atención de las personas, como la atención posdiagnóstico; c) Garantía de calidad, que asegura que las prestaciones seguirán patrones de exigencia técnica preestablecidos y construidos a partir de pruebas de evidencia médica, y d) Ga-

Finalmente, en cuarto lugar, resulta fundamental recordar que la única resolución que el Sistema Interamericano de protección de Derechos Humanos habría adoptado en interpretación del artículo $4^{\circ}$ número 1 de la "Convención Americana sobre Derechos Humanos" fue la Resolución del año 1981 No 23/81, en el Caso 2141 de la Comisión Interamericana de Derechos Humanos llamado "Baby Boy", donde se consideró que una interpretación restrictiva del artículo $4^{\circ}$ afectaría negativamente a la mayoría de los Estados americanos que permitían el aborto. En otras palabras, la frase "en general" contenida en el artículo $4^{\circ}$ № 1 del Pacto de San José de Costa Rica, se incorporó con el fin de rechazar, explícitamente, una interpretación como la que hace el Tribunal Constitucional chileno. Es decir, la norma sólo puede interpretarse, según la Comisión Interamericana de Derechos Humanos, de modo de hacerla armónica con la realidad legal de la mayoría de los países de la región que no consideran la protección de la vida del embrión de manera absoluta sino que, cosa distinta, han despenalizado el aborto en determinadas circunstancias.

${ }^{46}$ Mensaje presidencial No 1-347 del Proyecto de Ley que establece un Régimen de Garantías en Salud de 22 de mayo de 2002. No Boletín: 2947-11. Destacado agregado. 
rantía de cobertura financiera, en virtud de la cual el pago de las prestaciones no será un obstáculo para recibir los servicios asociados al sistema AUGE. Además, dispone financiamiento fiscal para la atención de salud de los carentes de ingresos $\mathrm{o}$ indigentes y establece la obligatoriedad de cotizar para salud de los trabajadores independientes.

Ello permite reconocer en la norma constitucional que se refiere al derecho a la protección de la salud, un contenido específico y mínimo, proporcionado por la nueva ley, la cual contempla garantías explícitas para un conjunto priorizado de prestaciones de salud en términos de acceso, calidad, oportunidad y costo. Se establece un estándar básico para la atención de salud de las enfermedades prioritarias -con un tiempo máximo para recibir atención y un desembolso financiero acotado para los pacientes- que constituye el núcleo del derecho constitucional a la salud reconocido por la Carta Fundamental. En cumplimiento con la obligación que se genera del derecho establecido en el artículo $19 \mathrm{~N}^{\circ} 9$ de la Constitución, hoy los prestadores de servicios sanitarios deben ofrecer a todos sus beneficiarios un "plan de salud mínimo" construido sobre la base de ciertas prioridades sanitarias, con garantías explícitas, cuya infracción puede denunciarse a la Superintendencia de Salud. De este modo, el núcleo básico del derecho a cuidado sanitario se garantiza "a todas las personas", sin discriminación y en condiciones de igualdad (recordemos que AUGE significa Acceso Universal a Garantías Explícitas). Por tanto, podemos concluir que a consecuencia de esta reforma legal, existe hoy un derecho a la protección de la salud constitucional y legalmente determinado.

Sin embargo lo anterior, no podemos obviar el hecho de que al momento de especificarse las condiciones y requisitos para ser beneficiario del sistema, por medio de los reglamentos y Guías Clínicas respectivas de cada una de las patologías AUGE, la igualdad e universalidad prometida por la Reforma se fue disolviendo. En efecto, si bien la estructura GES (Garantías Explícitas en Salud) apuntaba eficazmente a la reducción de barreras financieras insalvables para la mayor parte de la población, al momento de su implementación afloraron serios problemas derivados de la incorporación de técnicas de racionamiento sanitario de tipo "costobeneficio" propios de la economía de la salud, como por ejemplo, la exclusión de pacientes por su edad o su lugar de residencia, lo que es claramente incompatible con las obligaciones constitucionales e internacionales del Estado de Chile.

Entre los casos en que los criterios de racionamiento del "utilitarismo sanitario" 47 se aprecian con claridad destaca, en primer lugar, la garantía de analgesia en el parto, que sólo es accesible en la medida en que la mujer viva cerca de un centro

\footnotetext{
${ }^{47}$ Sobre las propuestas y dilemas éticos derivados del utilitarismo sanitario, véase ZúNIGA F., Alejandra (2008b) pp. 176-184.
} 
hospitalario dotado de especialistas anestesistas, lo que ocurre en aproximadamente un 30\% de los casos. Lo mismo sucede con las garantías para la artrosis de cadera, que excluye de atención a las personas menores de 55 años. También se produce la exclusión de pacientes frente al cáncer cervicouterino, cuyo tratamiento sólo es accesible en ciertas regiones del país, al igual que las garantías de prevención de este mismo cáncer, que se entrega sólo cada 3 años a mujeres de entre 25 a 64 años. Los exámenes preventivos del cáncer de mama, por su parte, también se proporcionan únicamente cada tres años y en el grupo etario de entre 50 y 54 años. Finalmente, el cáncer de vesícula -principal causa de muerte por cáncer en la mujer chilena- sólo incluye como beneficiarios a personas de entre los 35 y 49 años. No deja de llamar la atención el hecho de que estas enfermedades propiamente femeninas tengan coberturas tan restringidas, pues el AUGE pretendía precisamente reducir las desigualdades sociales y sanitarias generadas en razón del sexo. ${ }^{48}$ ¿Cómo afectan estas exclusiones de la administración a la comprensión del principio de igualdad y de supremacía constitucional?

La doctrina de Alexy permite recordar que los efectos materiales de la supremacía constitucional, que deben ser analizados tanto a nivel infraconstitucional como constitucional, exigen, a nivel infraconstitucional, una supremacía formal y también material de la constitución, lo que se traduce en que el contenido de las normas infraconstitucionales debe ser coherente con el de la Constitución. Esta coherencia se manifiesta tanto en sentido negativo como en sentido positivo. En sentido negativo, la supremacía material de la Constitución determina que ciertos contenidos incluidos en una norma infraconstitucional son inadmisibles. De igual forma, en sentido positivo, la supremacía material de la Constitución exige ciertos contenidos para las normas infraconstitucionales. En síntesis, la supremacía constitucional en sentido material prohíbe o rechaza ciertos contenidos y exige o impone otros. En tal sentido, se dice que la Constitución determina el contenido posible del sistema jurídico ${ }^{49}$.

¿Cómo podemos aplicar este análisis al caso del derecho a la protección de la salud? En primer lugar, es necesario reiterar que después del AUGE, en Chile existe un derecho a la protección de la salud, reconocido constitucionalmente y legalmente delimitado. Luego, la pregunta a resolver es la siguiente: cuáles son los límites negativos impuestos por la Constitución -y que por ende, están reservados al poder constituyente- $\mathrm{y}$ la legítima aspiración de la administración de determinar el llamado minimo sanitario, dentro del marco constitucionalmente admisible. Dicho de otro modo, ¿puede la administración infringir el principio de no discri-

${ }^{48}$ Véase, ZúNIIGA F., Alejandra (2011).

${ }^{49}$ AleXY, 2002. 
minación para efectos de determinar el mínimo sanitario? La respuesta claramente debe ser no. Los límites naturales que debiera tener toda legislación, a la luz de lo normado por la Constitución, son precisamente el respeto del principio de igualdad. Esto significa que una vez determinado el mínimo sanitario, su cobertura no podrá restringirse por la administración en razón de circunstancias como la edad, el sexo o el lugar de residencia. Ello constituye el límite negativo a la libertad de configuración que ha de tener la administración en la determinación del mínimo sanitario por razones técnicas ${ }^{50}$. Luego, resulta fundamental velar por la coherencia entre aquello que debe ser decidido en sede legislativa y aquello que ha de decidirse en sede administrativa a propósito del caso particular del derecho a cuidado sanitario. La administración, al mantener en más del 50\% de las guías clínicas, criterios de racionamiento sanitario que suponen la exclusión de pacientes en razón de su edad o de su lugar de residencia, infringe violentamente el principio de no discriminación garantizado por la norma constitucional.

Así, por lo demás, lo ha reconocido la reciente jurisprudencia de la Corte $\mathrm{Su}$ prema en el caso de Luis Vivanco Garabito contra el Hospital Hernán Henríquez Aravena de Temuco, donde sostuvo que el derecho a la protección de la salud goza de tutela normativa y que si bien éste no está garantizado por la acción cautelar contemplada en el artículo 20, "lo cierto es que los primeros incisos del numeral noveno del artículo 19 de la Carta Fundamental establecen criterios normativos rectores que el constituyente entrega al Estado, lo cual lleva a interpretar de modo extensivo la garantía constitucional" (...). Por tanto, concluye: "Si bien es cierto el tratamiento en cuestión se refiere a personas de 55 años y más, no por ello puede quedar sin recibir atención médica una persona por tener una edad inferior a ella, lo que apunta a un daño a su integridad, no sólo física sino psicológica y a su dignidad como ser humano, protegida en el artículo $1^{\circ}$ de la Constitución Política (...) Quien ha intentado esta acción cautelar le asiste el derecho igualitario, establecido por la ley y garantizado en la Constitución Politica, de acceder al tratamiento médico que le ha sido prescrito y, a su vez, la privación -por quien tiene a su cargo tal obligación- del derecho a ser intervenido quirúrgicamente, cuyo

\footnotetext{
${ }^{50}$ Aun cuando se trata de una reforma que tiene importantes consecuencias para el derecho garantido por la Constitución, se trata de una legislación que mantuvo vigente un sistema sanitario de tipo pluralista o mixto de consecuencias financieras claramente regresivas, pues las diferencias de atención entre quienes poseen y no poseen recursos todavía es muy importante. Las restricciones dispuestas por el sistema de prioridades sanitarias, sumado a los altos costos de la medicina privada, no permiten solucionar los problemas de equidad existentes. Para ello, era necesario ampliar la reforma y aprobar el primer proyecto AUGE que se presentó en el Congreso y que pretendía establecer un Fondo Solidario de Salud que accediera a traspasar recursos del sistema privado al sistema público de salud, logrando un efecto similar al de los sistemas sanitarios públicos universales y gratuitos, como el sistema canadiense o el sistema inglés. Véase, ZúNigA F., Alejandra (2007).
} 
único fin es propender la recuperación de la patología que le aqueja, importa una vulneración a la garantía establecida en la Constitución de la República, desde que es claro que la actuación del recurrido, al retardar el desempeño requerido, dificulta el restablecimiento de la salud del recurrente de protección, afectando su integridad física y síquica" ${ }^{1}$.

De este modo, la Corte Suprema reconoce la interpretación post-AUGE de la norma constitucional que se ha presentado más arriba. Esto es, acierta en afirmar que ahora el derecho a la protección de la salud tiene un contenido claro, definido por la ley, que no puede ser limitado por medio de decretos que pretendan, por la vía de las guías clínicas, restringir el acceso a las garantías GES sólo para algunas personas, infringiendo el principio de igualdad.

En la misma línea, este tribunal argumenta en el caso de Elena Vásquez Ibáñez contra FONASA, en el que se reconoce que: "Es deber de los órganos del Estado respetar y promover los derechos esenciales que emanan de la naturaleza humana y FONASA, como servicio público, presta un servicio de salud a la comunidad, por lo que un eventual afán de lucro o de autofinanciamiento, no puede llegar a constituir un objetivo. Por el contrario, debe velar por el efectivo cumplimiento de los derechos fundamentales que la Constitución Política de la República asegura a las personas en el artículo $19 \mathrm{~N}^{\circ} 1$ y 9 , esto es, el derecho a la vida e integridad física y el derecho a la protección de la salud, resaltando los perentorios términos y contenido de esta última disposición constitucional. En este marco, no debe olvidarse lo previsto en el artículo $6^{\circ}$ de la Carta Política, en cuyos incisos $1^{\circ}$ y $2^{\circ}$ consagra el principio de la supremacía constitucional sobre todas las demás normas que integran el ordenamiento jurídico positivo, lo que impide absolutamente que normas de inferior jerarquía a la Constitución, pudieran dejar sin aplicación una garantía que ella ampara y reconoce".

Continúa el tribunal argumentando: "Que si bien, esta última garantía, del número 9, no se encuentra amparada por el recurso de protección según lo establece el artículo 20, sí constituye un derecho garantizado a todas las personas por la Carta, por lo que no es posible desentenderse de su existencia para una adecuada administración de justicia". Por lo anterior, concluye la Corte Suprema: "el deber del Estado de Chile en materia de salud y el efectivo acatamiento de las garantías constitucionales de los derechos a la vida, integridad fisica, protección de la salud, independiente de si las normas reglamentarias contemplan o no el medicamento indicado como uno de aquellos que pueda utilizarse en el tratamiento del cáncer, obligan a proporcionar los medios para procurar el tantas veces citado medicamento, toda vez

${ }^{51}$ Corte Suprema, "José Luis Vivanco Garabito con Hospital Hernán Henríquez Aravena de Temuco", sentencia de 25/02/2010. Rol: 1324-2010. Destacado agregado. 
que lo que se está resolviendo es la mayor o menor sobrevida que pueda tener la recurrente, por lo que no le merece duda a esta Corte que debe proporcionársele el medicamento Herceptin, en cuanto le va a permitir sobrellevar el grave cáncer que la afecta en mejores condiciones, aspirando a una sobrevida mayor" 52 . Se obliga, de este modo, al órgano público a proporcionar la medicación solicitada por la recurrente para el tratamiento de su enfermedad AUGE, aun cuando la Guía Clínica respectiva no la contemple. Dicho de otro modo, la normativa del órgano administrativo que pretende, por la vía del decreto, restringir el acceso al derecho a la protección de la salud, sería inconstitucional pues contraría el derecho a la vida, igualdad y a la protección de la salud de la recurrente.

Finalmente, el Tribunal Constitucional ha reconocido, en sus recientes sentencias, que el derecho a la protección de la salud -que forma parte de los derechos sociales, llamados también derechos de prestación- son tales y no simples declamaciones o meras expectativas, cuya materialización efectiva quede suspendida hasta que las disponibilidades presupuestarias del Estado puedan llevarlos a la práctica. Que si bien la doctrina y jurisprudencia comparadas han señalado que los derechos sociales requieren la concretización legal de su contenido, también han realzado que la Constitución establece, en relación con ellos, un núcleo esencial, indisponible por el legislador. En esa línea, el propio tribunal ha enfatizado que el derecho a la protección de la salud, en cuanto derecho social, se halla sustancialmente ligado a otros atributos esenciales, como v. gr., el derecho a la vida y a la integridad tanto fisica como psíquica, todos los cuales deben ser tutelados y promovidos para infundir al ordenamiento legitimidad. "El Estado debe velar, como se lo exige la Constitución, por la vida de las personas. Lo hace directamente a través de su poder público para cautelarla de acciones de terceros y reconoce el derecho a la protección de la salud conforme al artículo 19, $\mathrm{N}^{\circ}$ 9, con el objeto de que, en caso de enfermedades, se preserven sus vidas" ${ }^{\prime 3}$.

En el mismo sentido, la sentencia del Tribunal Constitucional de 6 de agosto de 2010, Rol 1710, reconoce la existencia y plena vigencia, no sólo de derechos 'clásicos' (como el derecho a la igualdad ante la ley, donde se incluye expresamente el derecho de las mujeres a no sufrir discriminación en el acceso a servicios de atención médica y, en particular, en el establecimiento de las primas y las prestaciones de seguros, disponiéndose que, "en cualquier caso, los costes relacionados con el embarazo y la maternidad no deberán crear diferencias en

52 Corte Suprema, "Elena Vásquez Ibáñez con FONASA", sentencia de 28/01/2010. Rol: 9317-2009. Destacado agregado.

${ }^{53}$ Tribunal Constitucional, sentencia Rol: 976, 26 de junio de 2008. 
las primas o prestaciones" ${ }^{44}$ ), sino también el derecho a la protección de la salud y el derecho a la seguridad social. En esa línea, se establece que "los principales derechos sociales que la Constitución asegura a todas las personas son configurados a partir de la posibilidad de acceder a una determinada prestación. Así sucede con el derecho a la protección de la salud, en que se debe proteger 'el libre e igualitario acceso a las acciones' (artículo 19, $\mathrm{N}^{\circ}$ 9o); lo mismo sucede con el derecho a la seguridad social, en que la acción del Estado debe estar dirigida 'a garantizar el acceso de todos los habitantes al goce de prestaciones' (artículo 19, No 18)" ${ }^{\circ}$. Y continúa sosteniendo: "Que nuestra Constitución, a su vez, establece claramente que las prestaciones que envuelven los derechos en cuestión han de ser igualitarias y suficientes, sea que se otorguen por el Estado o por agentes privados. Las expresiones que en tal sentido emplea el texto constitucional son: 'libre e igualitario acceso' (artículo 19, No 9o) y 'prestaciones básicas uniformes' (artículo 19, No 18)" 56 .

Finalmente, concluye el Tribunal Constitucional, "la Constitución ha previsto normativamente la unidad del sistema de protección en materia de seguridad social. Dicha unidad está dada por el deber del Estado de 'garantizar' el goce del derecho a la protección de la salud y a la seguridad social, sea que lo preste por sí mismo o por sujetos privados (artículo 19, No 9, inciso cuarto, y No 18, inciso tercero)" 57 . Por tanto, "como se señaló por este Tribunal en la sentencia Rol 1287, la definición de la seguridad social ha encontrado sustancioso tratamiento en la doctrina chilena, la que ha profundizado en el detalle de sus componentes esenciales y en el alcance de los mismos. Así entendida como 'el conjunto de principios que reconocen a todo ser humano el derecho a los bienes indispensables para prevenir sus contingencias sociales y cubrir sus efectos y que regulan las instituciones requeridas para ello' (...) la seguridad social moderna se sustenta en determinados principios que le otorgan su esencia y que la reciente doctrina nacional ha enumerado como los siguientes: i) respecto de los sujetos amparados: universalidad subjetiva; ii) respecto de las contingencias cubiertas: universalidad objetiva; iii) respecto de las prestaciones: igualdad, integridad o suficiencia; iv) respecto del financiamiento: solidaridad, y v) respecto de la administración: unidad o descentralización, subsidiariedad"58.

\footnotetext{
${ }^{54}$ Tribunal Constitucional, sentencia Rol 976, considerando centésimo tercero y siguientes.

55 Tribunal Constitucional, sentencia Rol 976, considerando centésimo decimocuarto.

${ }^{56}$ Tribunal Constitucional, sentencia Rol 976, considerando centésimo trigésimo cuarto.

${ }^{57}$ Tribunal Constitucional, sentencia Rol 976, considerando centésimo trigésimo quinto.

58 Tribunal Constitucional, sentencia Rol 976, considerando centésimo trigésimo octavo.
} 
De este modo, como puede observarse, a diferencia de lo que ocurría en el pasado, la reciente jurisprudencia de los tribunales superiores de justicia y del Tribunal Constitucional, permite sustentar positivamente la tesis que se ha venido defendiendo: que el derecho a la protección de la salud posee hoy, después de la Reforma AUGE, un contenido legal claramente delimitado y susceptible de ser revisado a la luz de la normativa constitucional que reconoce, no sólo la existencia del derecho, sino que la necesidad de garantizar a todos el acceso a las prestaciones que de él se derivan en condiciones de igualdad.

\section{CONCLUSIÓN}

Antes de la reforma del AUGE, la jurisprudencia nacional, en materia de derecho a la vida y su relación con el derecho a la protección de la salud, no había construido aún una teoría coherente que permitiera una aplicación igualitaria de la garantía constitucional a los distintos casos que se le presentaban. En efecto, la jurisprudencia de los tribunales superiores de justicia era contradictoria y confusa, toda vez que las demandas de protección por acciones u omisiones que atentan contra la vida de las personas se resolvían, en general (como en los casos de los testigos de Jehová, las personas con VIH-Sida, los recurrentes para tratamientos de hemodiálisis y las huelgas de hambre), amparando el derecho, siempre y cuando ello no signifique gastos económicos importantes para el Estado.

Después de la referida Reforma Sanitaria, la jurisprudencia tanto de la Corte Suprema como del Tribunal Constitucional permite defender la tesis de que nuestra Constitución y legislación común adhiere a los postulados del liberalismo igualitario apuntando a garantizar, de modo universal, un mínimo sanitario decente. Ello se concluye de las sentencias relatadas y de la norma constitucional que reconoce el derecho a la protección de la salud y que hoy poseería un nuevo contenido que armoniza con las obligaciones que se generan como contrapartida al derecho establecido en el artículo $19 N^{\circ} 9$ de la Constitución. El Poder Legislativo, por medio de la Reforma Sanitaria, al fin habría especificado tanto el núcleo del derecho (el "mínimo sanitario decente" conformado por las enfermedades GES vigentes) como sus garantías asociadas.

Si bien antes del AUGE la norma constitucional era vista como meramente programática, al no disponer de un contenido claramente definido ni de mecanismo alguno de protección (puesto que la ley establecía que toda necesidad sanitaria se atendería con los recursos físicos y humanos de que se dispongan), en la actualidad es posible sostener que las GES -garantías explícitas de acceso, calidad, oportunidad y costo- para el conjunto priorizado de prestaciones sanitarias predefinidas, constituyen el núcleo del derecho constitucional a la salud recono- 
cido por la Carta Fundamental, y que dicho derecho debe, además, asegurarse sin discriminación.

\section{BiBLIOGRAFÍA CITADA}

1. ABRAMOVICH y Courtis: Los derechos sociales como derechos exigibles (Madrid, Trotta, 2002).

2. AleXY, Robert: Teoría de los derechos fundamentales (Madrid, Centro de Estudios Políticos y Constitucionales, 2002).

3. Bidart Campos: El Derecho de la Constitución y su Fuerza Normativa (Buenos Aires, Ediar, 1995).

4. Cea, José Luis (1999): El Sistema Constitucional de Chile, Sintesis Crítica (Valdivia, Universidad Austral de Chile).

5. Daniels, Norman: Just Health Care, Cambridge University Press, New York, 1985.

6. Diez, Sergio (1999): Personas y valores. Su protección constitucional (Santiago, Editorial Jurídica de Chile).

7. Dworkin, Ronald (1989), Los derechos en serio (Barcelona, Editorial Ariel).

8. FigueroA, Rodolfo: "Concepto de derecho a la vida", Ius et Praxis, año 14 $\mathrm{N}^{\circ} 1,2008$.

9. Nogueira, Humberto: "Análisis de la sentencia del tribunal constitucional chileno sobre el decreto supremo que regula la distribución de la píldora del día después (Levonorgestrel 0.75 MG)", Revista de Estudios Constitucionales, año $6 \mathrm{~N}^{\circ} 1,2008$.

10. Nogueira, Humberto (2009): “Los derechos económicos, sociales y culturales como derechos fundamentales efectivos en el constitucionalismo democrático latinoamericano", en Revista de Estudios Constitucionales, año 7 № 2, 2009.

11. Organización Mundial de la Salud: Estadísticas Sanitarias Mundiales 2009, OMS, Ginebra, 2009.

12. Organización Mundial de la Salud: Informe sobre la Salud en el Mundo 2000: Mejorar el desempeño de los sistemas de salud, OMS, Ginebra, 2000.

13. Peña, Carlos (1992): "Sobre el concepto y fundamento de los Derechos Humanos", en Revista Derecho y Humanidades, Volumen I (Santiago, Universidad de Chile).

14. Pfeffer, Emilio (1985): Manual de Derecho Constitucional, Tomo I (Santiago, Editorial Jurídica Ediar-Conosur).

15. RaWLs, John (1971): Theory of Justice (Oxford, Oxford University Press). 
16. Thomson, J. J. (1980): Defensa del Aborto, en Dworkin, Ronald, compilador, Filosofía del Derecho (Traducción de Javier Sáinz de los Terreros, México, Fondo de Cultura Económica).

17. TÓRTORA, Hugo: "El derecho a la vida en la jurisprudencia constitucional: aproximación al análisis de su delimitación, limitación y configuración", Revista de Estudios Constitucionales, año $3 \mathrm{~N}^{\circ}$ 2, 2005.

18. Urzúa Valenzuela (1991): Manual de Derecho Constitucional (Santiago. Editorial Jurídica de Chile).

19. Verdugo, Pfeffer y Nogueira (1994): Derecho Constitucional, Tomo I (Santiago, Editorial Jurídica de Chile).

20. VillaVicencio, Luis (2008): “¿Derechos humanos para quiénes? Reflexiones sobre algunas cuestiones embarazosas", en Revista de Derecho (Valdivia), diciembre 2008, volumen XXI, No 2 .

21. Villavicencio, Luis (2010): “¿Derechos básicos para quiénes?”, en Anuario de Filosofía Jurídica y Social ( $\left.{ }^{\circ} 27\right)$.

22. Zagrebelsky, Gustavo (2002): El Derecho Dúctil (Madrid, Editorial Trotta).

23. ZÚNiga F., Alejandra (2007): “Los Sistemas Sanitarios y el AUGE”, en Revista Acta Bioethica de la OPS/OMS (Vol. 13, No 2).

24. ZúNíga F., Alejandra (2008a): "Derechos del paciente y eutanasia en Chile", en Revista de Derecho de la Universidad Austral de Chile, Vol. XXI № 2.

25. ZÚNIIGA F., Alejandra (2008b): "Entre la igualdad y la eficiencia en materia sanitaria", en Revista Acta Bioethica de la OPS/OMS (Vol. 14, No 2).

26. ZúNíga F., Alejandra (2011): "Justicia y racionamiento sanitario en el Plan AUGE: Dilemas bioéticos asociados a la distribución de recursos escasos", en Revista Acta Bioethica $(2011 ; 17,1)$.

\section{JURISPRUDENCIA CITADA}

1. Corte Interamericana de Derechos Humanos, Caso Velásquez Rodríguez. Sentencia del 29 de julio de 1988. Serie C No 4. Párr. 164.

2. Corte Suprema, Rol N ${ }^{\circ} 4408-00$. Santiago, trece de diciembre del año 2000.

3. Revista Fallos del Mes, № 415, Santiago, 1993, p. 360.

4. Revista Gaceta Jurídica, No 59, Santiago, 1985, pp. 46-49.

5. Revista Fallos del Mes, № 402, Santiago, 1992, pp. 227-231.

6. Revista Gaceta Jurídica, No 232, Santiago, 1999, pp. 107-108.

7. Corte Suprema, Rol No 33.945, 18 de enero de 1996. 
8. Revista Gaceta Jurídica, No 50, Santiago, 1984, pp. 76-79.

9. Revista Gaceta Jurídica, No 101, Santiago, 1988, pp. 21-22.

10. Sentencia Corte Suprema, Rol No 33.945, 18 de enero de 1996.

11. Revista Gaceta Jurídica, No 91, Santiago, 1998, pp. 11-14.

12. Corte de Apelaciones, Rol No 3.025-2.001. Sentencia de 28 de agosto de 2001.

13. Corte Suprema, Rol No 3.599-2001, recogiendo el considerando No 8 del fallo de la Corte de Apelaciones.

14. Tribunal Constitucional de Chile, 18 de abril de 2008, Rol No 740.

15. Corte Suprema, sentencia de 25/02/2010. Rol No 1324-2010.

16. Corte Suprema, sentencia de 28/01/2010. Rol No 9317-2009.

17. Tribunal Constitucional de Chile, 26 de junio de 2008, Rol No 976.

18. Tribunal Constitucional de Chile, 6 de agosto de 2010, Rol No 1710. 
